\title{
Electrical bioimpedance spectroscopy in time-variant systems: Is undersampling always a problem?
}

\author{
Farhad Abtahi ${ }^{1}$, Fernando Seoane ${ }^{1,2}$ and Kaj Lindecrantz ${ }^{1,3}$ \\ 1. School of Technology and Health, KTH Royal Institute of Technology, 14152 Stockholm, Sweden \\ 2. School of Engineering, University of Borås, 501 90, Borås, Sweden \\ 3. Department of Clinical Science, Intervention and Technology, Karolinska Institute, 14186 Stockholm, Sweden \\ 4. E-mail any correspondence to: farhad.abtahi@sth.kth.se
}

\begin{abstract}
During the last decades, Electrical Bioimpedance Spectroscopy (EBIS) has been applied mainly by using the frequency-sweep technique, across a range of many different applications. Traditionally, the tissue under study is considered to be timeinvariant and dynamic changes of tissue activity are ignored by treating the changes as a noise source. A new trend in EBIS is simultaneous electrical stimulation with several frequencies, through the application of a multi-sine, rectangular or other waveform. This method can provide measurements fast enough to sample dynamic changes of different tissues, such as myocard. This high sampling rate comes at a price of reduction in SNR and the increase in complexity of devices. Although the frequencysweep technique is often inadequate for monitoring the dynamic changes in a variant system, it can be used successfully in applications focused on the time-invariant or slowly-variant part of a system. However, in order to successfully use frequencysweep EBIS for monitoring time-variant systems, it is paramount to consider the effects of aliasing and especially the folding of higher frequencies, on the desired frequency e.g. DC level. This paper discusses sub-Nyquist sampling of thoracic EBIS measurements and its application in the case of monitoring pulmonary oedema. It is concluded that by considering aliasing, and with proper implementation of smoothing filters, as well as by using random sampling, frequency-sweep EBIS can be used for assessing time-invariant or slowly-variant properties of timevariant biological systems, even in the presence of aliasing. In general, undersampling is not always a problem, but does always require proper consideration.
\end{abstract}

Keywords: Electrical bioimpedance spectroscopy, Aliasing in thoracic impedance spectroscopy, frequency-sweep EBIS, Thoracic Impedance Measurements, sub-Nyquist sampling

\section{Introduction}

Today, it is considered a common practice to characterize biological materials by using Electrical Bioimpedance Spectroscopy (EBIS). During the last decade EBIS has been used to assess the state or composition of various types of tissue, with applications including Body Composition Analysis (BCA) [1], tissue characterization for skin cancer detection [2] and cerebral monitoring [3,4]. It has also been utilised for non-invasive assessment of dynamics in physiological processes, such as in impedance-based systems for respiratory monitoring [5,6] and even the monitoring of cell growth [7-9].
In the assessment of tissue state or body composition, the EBIS signal is considered to be time-invariant and hence any dynamic changes are essentially ignored and instead treated as noise or interference. This interference can then be removed, or rather reduced, through the averaging, i.e. lowpass filtering, of the impedance signal. In many cases this works sufficiently well. However, there are situations when the frequency of the interference is higher than half the rate at which the impedance values are estimated, meaning that the Nyquist sampling theorem requirement is not fulfilled. The classical approaches to avoiding aliasing are faster sampling and the use of anti-aliasing filter. However, as the impedance samples are the result of a digital estimation process, and not the $\mathrm{A} / \mathrm{D}$ conversion of an analogue signal, the use of an anti-aliasing filter prior to digitisation is not an option. Furthermore, there are also limitations on how fast the impedance values can be estimated. Bioimpedance measurement is an estimation process and therefore not considered pure sampling; nevertheless, the term impedance sampling will be used henceforth.

Traditionally, the impedance spectra have been estimated by using a frequency-sweep technique, i.e. sweeping through a range of different stimulation frequencies. A higher speed spectroscopy is achievable through the use of broadband stimulation waveforms, e.g. square wave, step function, chirp, multi-sine and pseudo random noise [10, 11]. However, depending on the design requirements of the measurement system, the estimation process can be limited by estimation method, number of spectroscopy frequencies, estimation of lower frequencies (longer time), power consumption, size and weight.

In existing devices used for tissue status assessment, the estimation time for a full spectrum is in the order of seconds. For instance, the ImpediMed SFB7 (ImpediMed, Ltd., Eight Mile Plains, Queensland, Australia) takes 2 seconds to estimate one impedance spectrum of 256 frequencies between $4 \mathrm{kHz}$ and $1000 \mathrm{kHz}$ (0.5 spectra per second). This means that a variation component of above $0.25 \mathrm{~Hz}$ in the measured system will cause aliasing; for example, when the change in impedance due to respiration has a rate of 18 breaths per minute ( 0.3 breaths per second). 


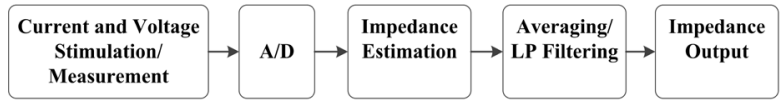

Figure 1: System for acquisition of impedance signals. Current stimulation and measurement could be any method; frequency sweep, multi-sine, or other.

The problem of impedance undersampling in real-time monitoring of myocardium tissue and the subsequent loss of important information and measurement artefacts that might destroy the EBIS measurement has been addressed by Sanchez et al. [12,13]. Sanchez et al. have recently introduced a new measuring approach based on multi-sine EBIS for time-varying bioimpedance monitoring [14]. This method and other multi-frequency excitation methods allows for higher sampling rates of the EBIS signal. However, this come at the price of a lower Signal to Noise Ratio (SNR) alongside other drawbacks described by Pliquett [15]. On the other hand, the existence of systemon-chip impedance measurement systems such as the AD5933 has established a good framework for developing compact and relatively low-cost EBIS devices upon the frequency-sweep method [16]. Therefore, the use of traditional frequency-sweep methods could still be effective in assessing the time-invariant or the slowly varying behaviour of a system, provided that proper consideration is given to high frequency interference. So what then is proper consideration? Is undersampling always a problem?

\section{Impedance acquisition process}

A general bioimpedance acquisition system is depicted in Figure 1. It is comprised of current/voltage stimulation and sensing, analogue to digital conversion, an impedance estimation procedure and a low-pass filter. The rate of A/D conversion has to be adapted to the frequency range of the stimulation current/voltage, which causes no problem. The problem addressed here is related to the time required for the impedance estimation, and the resulting rate at which impedance samples are produced.

The Nyquist-Shannon theorem (1928-1949) is fundamental to signal processing and information theory. It implies that a signal with a maximum frequency component of $f_{\max }$ has to be sampled at the rate of at least $2 f_{\max }$, for the information in the signal to be completely preserved [17, $18]$.

Sampling below the rate defined by the sampling theorem leads to folding, or aliasing, of image frequencies into the desired frequency band and therefore the original signal cannot be recovered (Figure 2). To avoid noise or interference above the frequencies of the signal becoming folded into the range of the signal, an anti-aliasing filter is normally introduced before the sampling. However, as the impedance samples are the result of a digital estimation process, in contrast to the $\mathrm{A} / \mathrm{D}$ conversion of the sensed

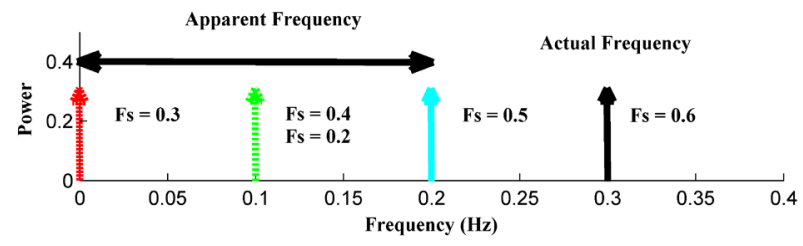

Figure 2: Apparent frequencies of a signal with a $0.3 \mathrm{~Hz}$ actual frequency component, sampled at $0.2,0.3,0.4$ and $0.5 \mathrm{~Hz}$ are depicted. It is clear that due to aliasing the apparent frequency differs from the actual frequency in all cases.

voltage/current, it is not possible at this stage to cope with potential aliasing through analogue filtering.

Irrespective of choice of estimation method for the impedance spectra, be it frequency sweep, multi-sine or other, longer estimation times yield better estimates; the more periods of the stimulation and sensing voltage/current, the higher signal-to-noise ratio (SNR). For slowly varying signals, e.g. body composition estimates, this would not constitute any conflict; there would be ample time to get a good SNR, if it were not for the influence from higher frequency noise/interference, e.g. respiratory influence on the impedance.

Many devices used for bioimpedance recording apply an intermediate impedance estimation time, i.e. neither a superfast method nor a very slow one. For estimation of very slowly varying parameters these devices instead average a number of estimates. It is important to note that the averaging of impedance samples is equivalent to one special case of LP filtering; more specifically moving average (MA) filtering with an nth order MA filter using equal coefficients, where $\mathrm{n}$ is the number of samples being averaged. The corresponding amplitude characteristic is depicted in Figure 3. There is, of course, nothing to say that other types of LP filters cannot be used, or could perhaps even perform better.

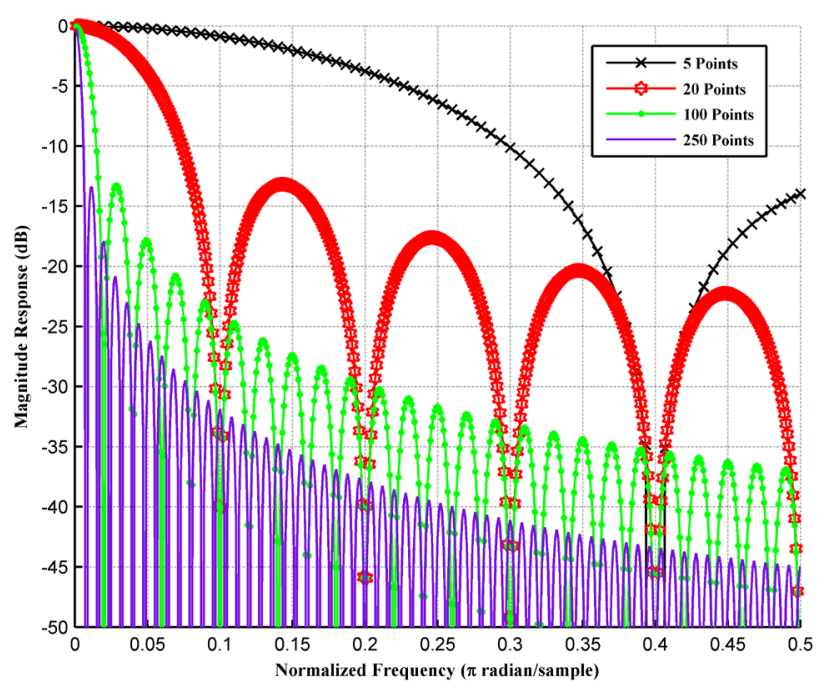

Figure 3: Frequency responses of 5, 20,100 and 250 points average filters are shown by black plus, red star, green circle and purple solid lines, respectively. 
How well averaging, or LP-filtering, performs in terms of increasing the signal-to noise ratio will depend highly on the frequency distribution of the noise. We can distinguish two general cases, narrow band interference and wide band interference.

\section{Narrowband interference}

Figure $4 a$ displays an example assuming narrowband interference; a relatively constant breathing rate of 15 breaths per minute $(0.25$ breaths per second) throughout the estimation time and an impedance sampling rate of 0.33 samples per second. In this example, the aliasing yields a spectrum around $0.08 \mathrm{~Hz}$ which can be suppressed through a long enough averaging time, i.e. a narrow enough LPfilter. Using a longer averaging time, by averaging over more samples, is equivalent to using a narrower LP filter (Figure 3). If time allows, an infinite attenuation of the interference can be achieved.

However, assuming an increase in interference frequency corresponding to a rate of 0.33 breaths per second (20 breaths per minute) the scenario changes drastically. Now the aliasing frequency coincides with $0 \mathrm{~Hz}$ (DC level or desired frequency for assessment in general), meaning that if the interference rate is perfectly constant then no averaging or LP filter will be able to even reduce the interference in the time-invariant impedance signal (Figure 4b).

\section{Wideband interference}

Let us now assume an interference pattern that does not produce a narrow band signal. The person may be talking, or, for any other reason, have a more random breathing rate (Figure 4c). In this case averaging will reduce the interference and the degree of attenuation of the interference will depend on the averaging time, i.e. the corresponding cut-off frequency of the LP filter, as well as the frequency distribution of the signal. Long averaging times yield lower residual interference and infinite averaging times will eliminate the interference entirely.

In summary; for relatively constant narrow high frequency interference, averaging may work very well or fail completely in reducing breathing interference from the time-invariant impedance signal, all depending on the ratio between impedance estimation time and interference frequency. For wideband interferences, averaging will always reduce the effect of aliasing and the degree of attenuation will depend upon the width of the frequency distribution of the interference and on the averaging time.

\section{Case Study - Pulmonary oedema}

Pulmonary oedema is the accumulation of fluid in the alveoli and parenchyma of the lungs and transthoracic impedance spectroscopy (TIS) is a potential method for monitoring pulmonary oedema. Since the accumulation of

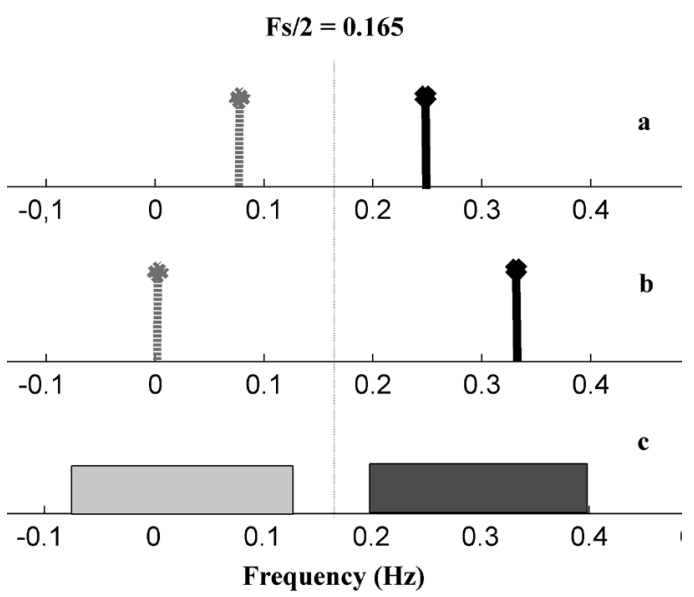

Figure 4: Actual and apparent frequencies due to aliasing for $0.25 \mathrm{~Hz}$ and $0.35 \mathrm{~Hz}$ narrow band signals and $(0.2-0.4 \mathrm{~Hz})$ wideband signals are depicted in plots a, b and c, respectively. Sampling frequency is considered to be $0.33 \mathrm{~Hz}$.

fluid slowly changes over time, frequency-sweep spectroscopy may be sufficient for this monitoring application. However, the basal TIS signal that indicates the degree of fluid accumulation will always be afflicted with a signal induced by the respiratory and cardiac activity. Table 1 , from Brown et al [19], shows that the breathing related part of the impedance is in the order of 5-8 percent of the basal impedance of interest for oedema monitoring, meaning that it can hardly be ignored. Cardiac activity however, is less than one percent, which in most cases is negligible. Thus, the respiration rate in relation to the sampling rate of the EBIS device becomes crucial. Table 2, from Lindh et al. [20], gives the normal breathing rate for humans at different ages.

It is clear that using an impedance device, such as SFB7, capable of providing an impedance spectrum in $2 \mathrm{~s}$, corresponding to an impedance sampling rate of $0.5 \mathrm{~Hz}$, will not guarantee fulfilment of the Nyquist criterion even for adults. However, the frequencies above $0.25 \mathrm{~Hz}$ (half of sampling rate) up to the maximum frequency of respiration in adults $(0.33 \mathrm{~Hz})$ will fold down into frequencies above $0.17 \mathrm{~Hz}$, and these frequency components will therefore be reduced by at least $20 \mathrm{~dB}$ by averaging 20 points, i.e. over $40 \mathrm{~s}$ (compare with Figure 3). Now with the original

Table 1: Thoracic impedance; mean data with the standard deviation for the 12 subjects.

\begin{tabular}{c|ccc}
$\begin{array}{c}\text { Frequency } \\
(\mathbf{k H z})\end{array}$ & $\begin{array}{c}\text { Impedance } \\
(\boldsymbol{\Omega}) \boldsymbol{Z}\end{array}$ & $\begin{array}{c}\text { Respiratory component } \\
(\boldsymbol{\Omega}) \mathbf{Z}_{\mathbf{r}}\end{array}$ & $\begin{array}{c}\text { Cardiac component } \\
(\mathbf{m} \boldsymbol{\Omega}) \mathbf{Z}_{\mathbf{c}}\end{array}$ \\
\hline $\mathbf{9 . 6}$ & $24.56 \pm 4.09$ & $1.68 \pm 0.43$ & $98.4 \pm 33.0$ \\
$\mathbf{1 9 . 2}$ & $23.93 \pm 4.10$ & $1.66 \pm 0.43$ & $89.1 \pm 32.5$ \\
$\mathbf{3 8 . 4}$ & $22.64 \pm 4.08$ & $1.62 \pm 0.42$ & $79.4 \pm 32.7$ \\
$\mathbf{7 6 . 8}$ & $21.04 \pm 3.96$ & $1.55 \pm 0.41$ & $63.5 \pm 26.2$ \\
$\mathbf{1 5 3 . 6}$ & $19.35 \pm 3.78$ & $1.47 \pm 0.39$ & $54.1 \pm 22.5$ \\
$\mathbf{3 0 7 . 2}$ & $17.81 \pm 3.50$ & $1.37 \pm 0.36$ & $40.4 \pm 17.9$ \\
$\mathbf{6 1 1 . 1}$ & $15.70 \pm 2.80$ & $1.10 \pm 0.30$ & $28.2 \pm 12.4$
\end{tabular}


Table 2: Normal respiratory rates at different ages

\begin{tabular}{|c|c|c|}
\hline & $\begin{array}{c}\text { RR } \\
\text { (Breath/Minute) }\end{array}$ & $\begin{array}{c}\text { Frequency } \\
(\mathrm{Hz})\end{array}$ \\
\hline Newborns & 44 & 0.73 \\
\hline Infants & $20-40$ & $0.33-0.66$ \\
\hline Children & $18-30$ & $0.30-0.5$ \\
\hline Adults & $12-20$ & $0.20-0.33$ \\
\hline
\end{tabular}

breathing signal, being one fifth of the basal impedance, reduced by a factor 10 , the influence of respiration, now $2 \%$, may be acceptable. If so, the sampling theorem is not fulfilled, but still works well.

For children, infants and neonates things are worse. For all these categories of young people a breathing rate of 30 breaths per minute, corresponding to a breathing frequency of $0.5 \mathrm{~Hz}$, is normal. This is particularly true for infants and neonates. With a sampling rate of $0.5 \mathrm{~Hz}$ for the impedance estimation process, the breathing interference will be aliased to $0 \mathrm{~Hz}$, and cannot be reduced by averaging or any other type of LP filtering. If the breathing rate is not perfectly fixed at $0.5 \mathrm{~Hz}$, which is seldom the case, the spectrum of the respiratory interference will be spread around $0.5 \mathrm{~Hz}$, and the corresponding aliased frequencies spread around $0 \mathrm{~Hz}$. However, the fact that the spectral content is not concentrated to $0 \mathrm{~Hz}$ exactly, means that the LP filter will reduce the interference; how much will depend on the spread of the spectrum and the bandwidth and delay of the LP filter. As the breathing pattern is unknown beforehand, the use of this low impedance estimation rate should be avoided with children. If it cannot be avoided, then an excessive averaging time may solve the problem. Long averaging times will give a narrow LP filter thus removing a lot of none-zero $\mathrm{Hz}$ interference and will also increase the probability that the breathing rate is not perfectly fixed throughout the epoch. Special care is definitely called for and particularly for children on ventilators, the fixed breathing rate must be considered thoroughly.

Other EBIS acquisition times will lead to different results, but the type of analysis will be the same. For instance, an EBIS acquisition time of $3 \mathrm{~s}$, corresponding to 0.33 samples per second, will be problematic for all age categories, as breathing above 10 breaths per minute $(0.166$ breaths per second) is a realistic breathing rate for all ages. As concluded earlier, this is the worst possible combination of breathing rate and sampling rate because averaging will yield no reduction in breathing interference at all. Speeding the acquisition up to $2 \mathrm{~s}(0.5$ samples/s $)$ means that breathing rates less than 15 breaths per minute $(0.25$ breaths per second) will fulfil the sampling theorem (see the change in the apparent frequency of the dash-circle light blue line for frequencies higher than $0.25 \mathrm{~Hz}$, Figure 5), but as concluded above, some aliasing may be tolerable. If we assume a maximum breathing rate of 25 breaths per minute ( 0.42 breaths per second), a maximum rate that will cover most cases of normal breathing in adults, its aliasing image will fold at 0.08 breaths/s (the $\mathrm{y}$ value of the dash-circle light blue line at $\mathrm{x}$ equal to $0.42 \mathrm{~Hz}$, Figure 5). If we want

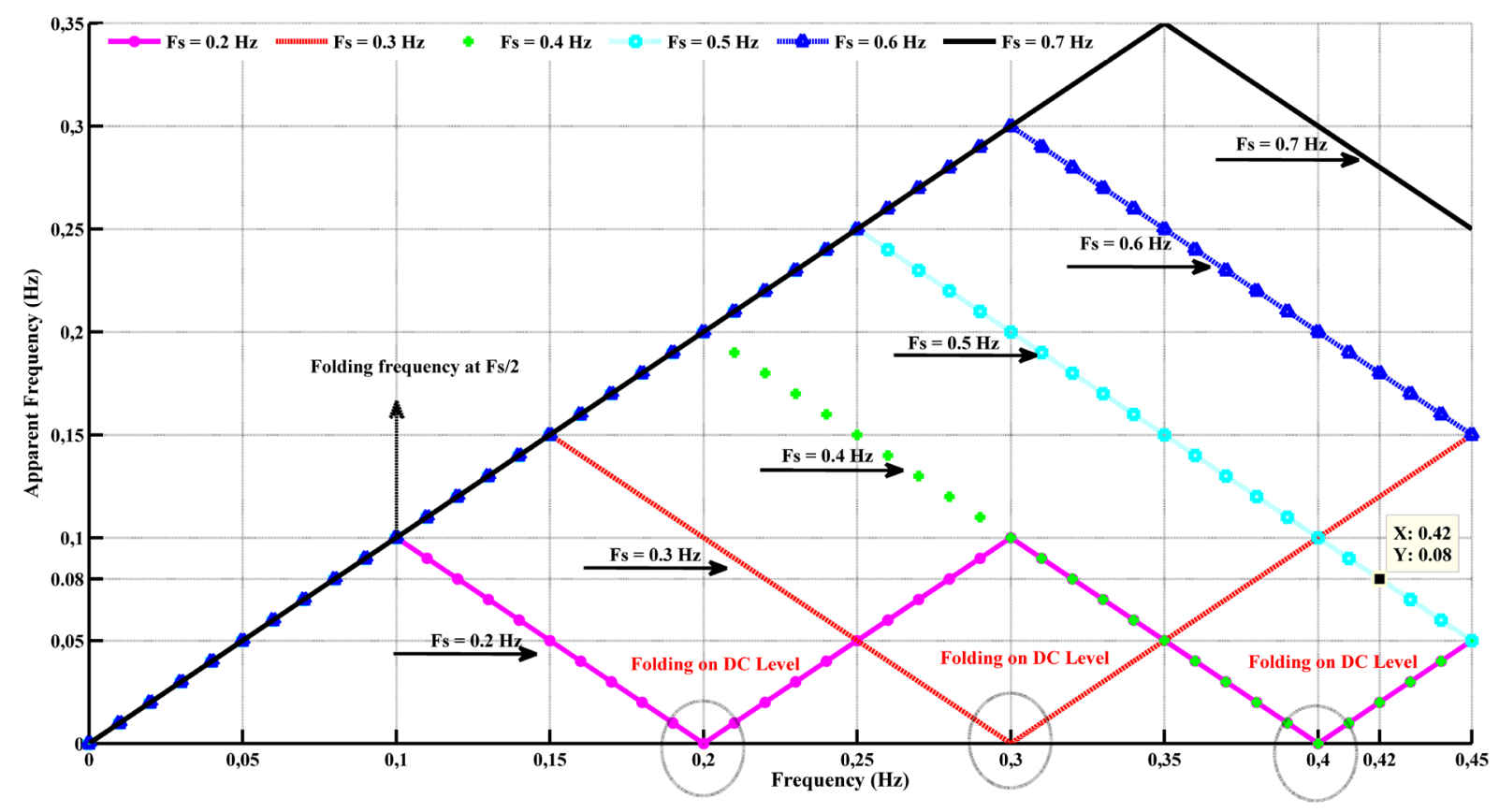

Figure 5: Actual and apparent frequencies at sampling frequencies are illustrated. It is obvious that apparent and original frequencies are the same when the sampling frequency is equal to the Nyquist frequency. In general all sampling frequencies less than the Nyquist rate are causing aliasing. However, for lower sampling rates, i.e. sampling at 0.2 and $0.3 \mathrm{~Hz}$, higher frequencies are folded into DC level and hence DC level cannot be recovered even by using the narrowest lowpass filter. Considering a maximum breathing rate of 25 breaths per minute $(0.42 \mathrm{~Hz})$ and sampling frequency of $0.5 \mathrm{~Hz}$, the maximum frequency component $(0.42 \mathrm{~Hz})$ is folding on $0.08 \mathrm{~Hz}$, as shown in the figure (dash-circle light blue line at $\mathrm{X}=0.42$ ). 
to reduce breathing related interference in the basal impedance by $10 \%$, we need averaging/LP-filtering that attenuates approximately $20 \mathrm{~dB}$ at $0.08 \mathrm{~Hz}(0.16$ of the sampling rate). In Figure 3 we can see that something in the order of 50 point averaging will be needed. Consequently, averaging over 100 seconds of the signal will give us the required reduction in breathing interference under the stated conditions.

\section{Discussion}

There is a general trend that more sensors, cameras etc. are being utilized in an ever wider range of applications. Consequently, demands to reduce resources (computational power, storage and time) needed for sampling and reconstruction of signals and images is increasing. In addition, the Nyquist rate of wideband analogue signals may exceed the specification of the best analogue-to-digital convertors (ADC). This has led to the development of the compressive sensing theorem, a novel sampling theorem proposing that signals can be sampled at a rate proportional to their information content, instead of their bandwidth. This theorem is based on random sampling of sparse signals, i.e. those that have only a few non-zero coefficients in any transform domain [21,22]. Random sampling is also used successfully with wideband signals in communication applications when following the sampling theorem may not always be possible [23,24]. In the future, these novel methods could permit us to sample time-variant biological systems with rates below those established in classical signal processing. However, in the framework discussed here, it is clear that having a sampling interval that is not fixed but random, for instance uniformly distributed, would solve the problem of a fixed breathing rate potentially yielding EBIS estimates where averaging does not remove the breathing interference.

A recent patent application from Philips [25] relating to an impedance based detection of lung oedema, suggests a step in the opposite direction. Rather than introducing random sampling, they propose synchronised sampling. The same problem is also addressed in [26]. The patent provides an example of frequency sweep estimation at a sampling rate of $0.33 \mathrm{~Hz}$. In parallel with the impedance estimation, a respiratory signal from a different source, e.g. an inductive belt, is recorded. Through modelling the influence of breathing in the impedance signal, the breathing interference can be compensated for. It is not explicitly stated but naturally the sampling of the separate breathing signal has to fulfil the sampling theorem. This method is somewhat more complex to implement as it requires separate access to information about the breathing cycle. Depending on how it is implemented there is also a slight risk that alterations in depth of breath are interpreted as changes in basal impedance. Also, as some degree of averaging, i.e. LP filtering, may still be needed for the removal of interferences and artefacts other than breathing, the advantages of this method are not obvious.

Another potential problem with thoracic EBIS measurements may originate from frequency-sweep step delay, the measurement time between two successive frequencies, which is introduced in [27]. However, in the case of pulmonary oedema, the effect of this delay will be suppressed by averaging and does not require additional attention.

The scope of this paper relates only to the relationship between the frequency content of an interfering signal and the impedance sampling rate and compromises within this context. The design of a system for acquisition of impedance spectra involves greater number of degrees of freedom, such as choice of estimation method, e.g. swept sine, multi sine, or other, and the number of frequencies from which the spectrum is estimated etc. More frequencies mean a better estimation of the spectrum, but at the price of longer estimation times. The mere fact that the signal in some cases cannot be considered stationary throughout the duration of the estimation may in itself be a problem. In this paper respiratory interference in basal impedance assessments is one example where a sampling rate compromise can be relevant, but as deduced from Tables 2 and 3, a similar approach may be of value for a respiratory signal with an interfering cardiac component.

\section{Conclusion}

In general, undersampling is not necessarily a problem but it always requires proper consideration. Future work, investigating the emerging field of compressed sensing methods, may produce new and better ways of acquiring electrical bioimpedance spectra.

\section{References}

1. van Marken Lichtenbelt WD, Westerterp KR, Wouters L, Luijendijk S. Validation of bioelectrical-impedance measurements as a method to estimate body-water compartments. The American Journal of Clinical Nutrition. 1994;60(2):159-66.

2. Aberg P, Nicander I, Hansson J, Geladi P, Holmgren U, Ollmar S. Skin cancer identification using multifrequency electrical impedance-a potential screening tool. IEEE Transactions on Biomedical Engineering. 2004;51(12):2097102. http://dx.doi.org/10.1109/TBME.2004.836523

3. Lingwood BE, Dunster KR, Healy GN, Ward LC, Colditz PB. Cerebral impedance and neurological outcome following a mild or severe hypoxic/ischemic episode in neonatal piglets. Brain Research. 2003;969(1):160-7. http://dx.doi.org/10.1016/S0006-8993(03)02295-9 
4. Seoane F, Lindecrantz K, Olsson T, Kjellmer I, Flisberg A, Bågenholm R. Spectroscopy study of the dynamics of the transencephalic electrical impedance in the perinatal brain during hypoxia. Physiological Measurement. 2005;26:849. http://dx.doi.org/10.1088/0967-3334/26/5/021

5. Freimark D, Arad M, Sokolover R, Zlochiver S, Abboud S. Monitoring lung fluid content in CHF patients under intravenous diuretics treatment using bio-impedance measurements. Physiological Measurement. 2007;28(7):269. http://dx.doi.org/10.1088/0967-3334/28/7/S20

6. Reiter H, Muehlsteff J, Sipila A, editors. Medical application and clinical validation for reliable and trustworthy physiological monitoring using functional textiles: Experience from the HeartCycle and MyHeart project. Engineering in Medicine and Biology Society, EMBC, 2011 Annual International Conference of the IEEE.

7. Cheng X, Liu Y-s, Irimia D, Demirci U, Yang L, Zamir L, et al. Cell detection and counting through cell lysate impedance spectroscopy in microfluidic devices. Lab on a Chip. 2007;7(6):746-55. http://dx.doi.org/10.1039/b705082h

8. Ehret R, Baumann W, Brischwein M, Schwinde A, Stegbauer $\mathrm{K}$, Wolf B. Monitoring of cellular behaviour by impedance measurements on interdigitated electrode structures. Biosensors and Bioelectronics. 1997;12(1):29-41. http://dx.doi.org/10.1016/0956-5663(96)89087-7

9. Soley A, Lecina M, Gamez X, Cairo J, Riu P, Rosell X, et al. On-line monitoring of yeast cell growth by impedance spectroscopy. Journal of Biotechnology. 2005;118(4):398-405. http://dx.doi.org/10.1016/j.jbiotec.2005.05.022

10. Sanchez B, Schoukens J, Bragos R, Vandersteen G. Novel estimation of the electrical bioimpedance using the local polynomial method. Application to in vivo real-time myocardium tissue impedance characterization during the cardiac cycle. IEEE Transactions on Biomedical Engineering. 2011;58(12):3376-85. http://dx.doi.org/10.1109/TBME.2011.2166116

11. Sanchez B, Vandersteen G, Rosell-Ferrer J, Cinca J, Bragos R, editors. In-cycle myocardium tissue electrical impedance monitoring using broadband impedance spectroscopy. Engineering in Medicine and Biology Society, EMBC, 2011 Annual International Conference of the IEEE.

12. Sanchez B, Schoukens J, Bragos R, Vandersteen G. Novel Estimation of the Electrical Bioimpedance using the Local Polynomial Method. Application to in-vivo real-time Myocardium Tissue Impedance Characterization during the Cardiac Cycle. IEEE Transactions on Biomedical Engineering. 2011;58(12):3376-3385. http://dx.doi.org/10.1109/TBME.2011.2166116

13. Sanchez B, Vandersteen G, Rosell-Ferrer J, Cinca J, Bragos R, editors. In-cycle myocardium tissue electrical impedance monitoring using broadband impedance spectroscopy. Conf Proc IEEE Eng Med Biol Soc. 2011;2011:2518-2521. http://dx.doi.org/10.1109/IEMBS.2011.6090697.
14. Sanchez B, Louarroudi E, Jorge E, Cinca J, Bragos R, Pintelon R. A new measuring and identification approach for timevarying bioimpedance using multisine electrical impedance spectroscopy. Physiological Measurement. 2013;34(3):339. http://dx.doi.org/10.1088/0967-3334/34/3/339

15. Pliquett $U$, editor. Time-domain based impedance measurement: strengths and drawbacks. J. Phys.: Conf. Ser. 434012092 http://dx.doi.org/10.1088/1742-6596/434/1/012092

16. Ferreira J, Seoane F, Lindecrantz K. AD5933-based electrical bioimpedance spectrometer. Towards textile-enabled applications. Engineering in Medicine and Biology Society, EMBC. 2011 Annual International Conference of the IEEE.

17. Nyquist H. Certain topics in telegraph transmission theory. Transactions of the American Institute of Electrical Engineers. 1928;47(2):617-44 http://dx.doi.org/10.1109/T-AIEE.1928.5055024

18. Shannon CE. Communication in the presence of noise. Proceedings of the IRE. 1949;37(1):10-21. http://dx.doi.org/10.1109/JRPROC.1949.232969

19. Brown BH, Barber DC, Morice A, Leathard AD. Cardiac and respiratory related electrical impedance changes in the human thorax. IEEE Transactions on Biomedical Engineering. 1994;41(8):729-34. http://dx.doi.org/10.1109/10.310088

20. Lindh W, Pooler M, Tamparo C, Dahl BM. Delmar's comprehensive medical assisting: administrative and clinical competencies: Cengage Learning; 2009.

21. Becker SR. Practical compressed sensing: modern data acquisition and signal processing: California Institute of Technology; 2011.

22. Donoho DL. Compressed sensing. IEEE Transactions on Information Theory. 2006;52(4):1289-306. http://dx.doi.org/10.1109/TIT.2006.871582

23. Laska J, Kirolos S, Massoud Y, Baraniuk R, Gilbert A, Iwen $\mathrm{M}$, et al., editors. Random sampling for analog-to-information conversion of wideband signals. 2006 IEEE Dallas/CAS Workshop on Design, Applications, Integration and Software.

24. Bilinskis I. Digital alias-free signal processing: John Wiley \& Sons; 2007. http://dx.doi.org/10.1002/9780470511633

25. Cuba-Gyllensten I, Lars IG, Bonomi AG, Riistama JM, Caffarel J, Reiter H, inventors; Koninklijke Philips Electronics N.V., assignee. Biompedance spectrography system and method. Patent WO 2013/121327 A1. 2013.

26. Cuba-Gyllensten I, Abtahi F, Bonomi A, Lindecrantz K, Seoane F, Amft O, editors. Removing respiratory artefacts from transthoracic bioimpedance spectroscopy measurements. Journal of Physics: Conference Series; 2013: IOP Publishing.

27. Abtahi F, Gyllensten IC, Lindecrantz K, Seoane F. Software Tool for Analysis of Breathing-Related Errors in Transthoracic Electrical Bioimpedance Spectroscopy Measurements. Journal of Physics: Conference Series. 2012;407(1):012028. 